EPJ Web of Conferences 38, 10001 (2012)

DOI: $10.1051 /$ epjconf/20123810001

(c) Owned by the authors, published by EDP Sciences, 2012

\title{
Progress on the study of nuclear states with mixed proton-neutron symmetry
}

\author{
N. Pietralla ${ }^{1,2, a}$, Th. Möller ${ }^{1}$, T. Ahn ${ }^{1,3,4}$, Ch. Bauer $^{1}$, L. Coquard ${ }^{1}$, M. Danchev ${ }^{5}$, K.A. Gladnishki ${ }^{5}$, A. Krugmann $^{1}$, J. \\ Leske $^{1}$, O. Möller ${ }^{1}$, P. von Neumann-Cosel ${ }^{1}$, V.Yu. Ponomarev ${ }^{1}$, G. Rainovski ${ }^{5}$, A. Scheikh Obeid ${ }^{1}$, M. Scheck ${ }^{1}$, Ch. $^{2}$ \\ Walz $^{1}$, and J. Wambach ${ }^{1,2}$ \\ 1 Institut für Kernphysik, Technische Universität Darmstadt, 64289 Darmstadt, Germany \\ 2 GSI Helmholtzzentrum für Schwerionenforschung mbH, 64291 Darmstadt, Germany \\ 3 Wright Nuclear Structure Laboratory, Yale University, New Haven, CT, U.S.A. \\ 4 National Superconducting Cyclotron Lab, Michigan State University, East Lansing, MI 48824, USA \\ 5 Faculty of Physics, St. Kliment Ohridski University of Sofia, BG-1164 Sofia, Bulgaria
}

\begin{abstract}
The one-quadrupole phonon excitation of mixed symmetry, the $2_{1, \mathrm{~ms}}^{+}$state, is a fundamental building block of nuclear structure. This article gives a summary of our recent experimental research on this excitation mode in the $A=90$ and $A=130$ mass regions.
\end{abstract}

\section{Introduction}

The nuclear proton-neutron degree of freedom and its fundamental impact on nuclear structure represents one of the central aspects of modern nuclear structure physics. The structure of collective nuclear excitations is determined by the nuclear shell structure that itself evolves as a function of proton and neutron numbers. The evolution of nuclear shell structure as a function of neutron-excess provides us with a sensitive test for modern effective nuclear interactions. It is studied at current radioactive ion beams by tracing, for instance, the simplest properties of low-energy quadrupole-collective states to the most neutron-rich nuclei accessible. The evolution of nuclear shells also dominates the formation of nuclear deformation and collectivity. It sets the conditions for the emergence of excited nuclear quantum states with wave functions that include many proton excitations and neutron excitations within the valence shell that collectively couple in phase. This inphase coupling of proton and neutron valence-shell excitations simultaneously leads to the occurrence of orthogonal nuclear valence-shell excitations with partial out-of-phase coupling of proton and neutron components. These states represent a closely related aspect of the nuclear protonneutron degree of freedom. Indeed, nuclear excitations may be classified according to the proton-neutron symmetry of their wave functions, either in terms of isospin in the framework of models that use nucleons as the fundamental degree of freedom or in terms of $F$-spin in approximate bosonic nuclear models for collective valence-shell excitations at low energies.

The formulation of the interacting boson model (IBM2 ) in its $F$-spin limit [1-3] has emphasized the fundamental

\footnotetext{
a e-mail: pietralla@ikp.tu-darmstadt.de
}

role of collective proton-neutron non-symmetric valenceshell excitations, for the first time. Nuclear excitations with $F$-spin quantum number $F<F_{\max }=\left(N_{\pi}+N_{v}\right) / 2$, where $N_{\pi(v)}$ is half the number of valence protons (neutrons), contain parts in their wave functions where proton bosons and neutron bosons are coupled antisymmetrically with respect to the exchange of proton and neutron labels. These states have been called mixed-symmetry states (MSSs). Prominent examples of MSSs are the $J^{\pi}=1^{+}$ scissors mode of deformed nuclei or the mixed-symmetry $2_{1, \mathrm{~ms}}^{+}$one-phonon vibration in heavy spherical nuclei. Information on the pro-ton-neutron symmetry of the lowenergy nuclear states, including the ground state, its collective excitations, and MSSs, is needed for a correct interpretation of the role of the nuclear proton-neutron degree of freedom in the formation of nuclear structure. After the discovery of the scissors mode [4] and the clarification of its quadrupole-collective character $[5,6]$ it became obvious that the isovector quadrupole excitation of the valence shell represents the building block of mixed-symmetric structures.

Vibrational nuclei exhibit a one-quadrupole phonon excitation as the lowest-lying state of mixed-pn symmetry, i.e the $2_{1, \mathrm{~ms}}^{+}$state. Its close relation to the $2_{1}^{+}$state is evident in the $Q$-phonon scheme [7], where the wave functions of the one-quadrupole phonon excitations are well approximated by the expressions

$$
\begin{aligned}
\left|2_{1}^{+}\right\rangle & \simeq Q_{s}\left|0_{1}^{+}\right\rangle=\left[Q_{\pi}+Q_{v}\right]\left|0_{1}^{+}\right\rangle \\
\left|2_{1, \mathrm{~ms}}^{+}\right\rangle \simeq Q_{m}\left|0_{1}^{+}\right\rangle & =N\left[\frac{Q_{\pi}}{N_{\pi}}-\frac{Q_{v}}{N_{v}}\right]\left|0_{1}^{+}\right\rangle .
\end{aligned}
$$

Here, $Q_{\pi, v}\left(N_{\pi, v}\right)$ denote the proton and neutron quadrupole operators (boson numbers), $N=N_{\pi}+N_{v}$, and $\left|0_{1}^{+}\right\rangle$is the (in general highly correlated) ground state of a collectively

This is an Open Access article distributed under the terms of the Creative Commons Attribution License 2.0, which permits unrestricted use, distribution, and reproduction in any medium, provided the original work is properly cited. 
vibrating even-even nucleus. Despite its fundamental role in nuclear structure, the $2_{1 \mathrm{~ms}}^{+}$state has only recently been studied systematically, [8-12]. The dominant fragments of the one-phonon $2_{1, \mathrm{~ms}}^{+}$state are observed at about $2 \mathrm{MeV}$ excitation energy. Due to their isovector character, MSSs decay rapidly by dipole transitions and are very short lived, typically a few tens of femtoseconds. Large $M 1$ matrix elements of $\approx 1 \mu_{N}$ are in fact the unique signatures for MSSs. Consequently, lifetime information is needed for making safe assignments of mixed symmetry to a nuclear energy level. A review article on the status of experimental information on mixed-symmetry states in vibrational nuclei has been published [13]. We report on our recent progress on this field.

\section{Experimental method}

Projectile-Coulomb excitation has been established as a powerful method for the identification and investigation of one-phonon MSSs [10]. After this approach has first been applied to the investigation of the $2_{1, \mathrm{~ms}}^{+}$state of ${ }^{96} \mathrm{Ru}$ at the Yale Tandem accelerator [10], we have initiated a research programme on the $2_{1, \mathrm{~ms}}^{+}$state at ANL with the nucleus ${ }^{138} \mathrm{Ce}$ as a case study [12]. Crucial influence of sub-shell closures on mixed-symmetry structures was observed [12], a phenomenon which sensitively tests the effective proton-neutron interaction in microscopic valence shell models [14]. The one-phonon $2_{1, \mathrm{~ms}}^{+}$state of ${ }^{136} \mathrm{Ce}$ has recently been identified from similar Coulomb-excitation experiments at Gammasphere [15].

A sequence of experiments has been performed at Argonne National Laboratory. The superconducting ATLAS accelerator provided the ion beams of stable even-even isotopes, e.g. of the Xenon, Barium and Cerium isotopic chains, with energies corresponding to $\sim 85 \%$ of the Coulomb barrier for a reaction on ${ }^{12} \mathrm{C}$ nuclei. The beam intensities amounted typically to $\sim 1 \mathrm{pnA}$. The beams were

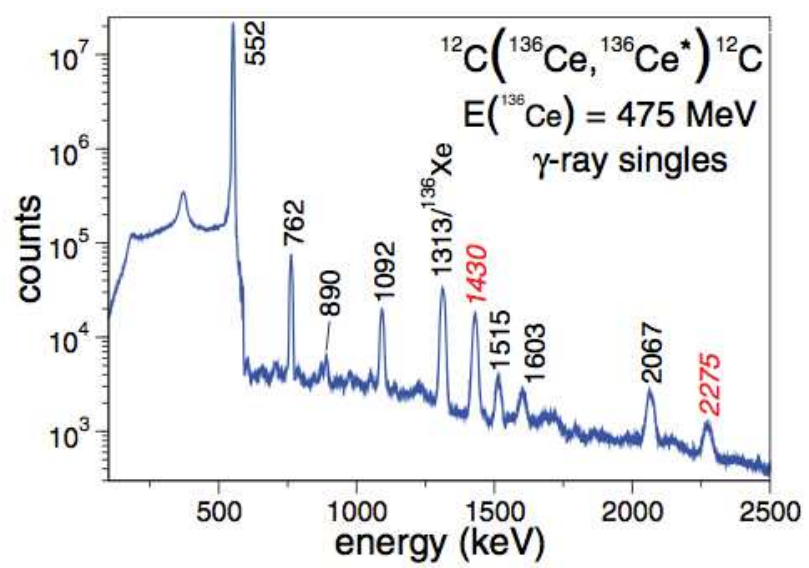

Fig. 1. Background-subtracted and Doppler-corrected singles $\gamma$ ray spectrum summed over all Ge detectors of the Gammasphere array at ANL after Coulomb excitation of ${ }^{136} \mathrm{Ce}$ on a carbon target [15]. impinging on a stationary carbon target of thickness 1 $\mathrm{mg} / \mathrm{cm}^{2}$. Light target ions were chosen in order to favor the one-step Coulomb excitation process over multi-step processes for ease of data evaluation. The $\gamma$-rays emitted by Coulomb-excited states of the beam nuclei were detected in the Gammasphere array which consists of $\sim 100$ high purity Compton suppressed Germanium detectors arranged in 16 rings $[16,17]$. An event was defined by a $\gamma$-ray of multiplicity 1 or higher. Doppler correction (recoiling velocity 6-8\%) and background subtraction (difference between the "in-beam" spectrum and the "off-beam" spectrum scaled to eliminate the $1461 \mathrm{keV}{ }^{40} \mathrm{~K}$ line) had been applied in order to obtain the total single spectra. Figure 1 displays data from the projectile-Coulomb excitation reactions of a ${ }^{136} \mathrm{Ce}$-ion beam on a carbon target.

The experimental $\gamma$-ray spectra are dominated by the decays of low-spin states, such as $2^{+}$or $3^{-}$states, that are predominantly populated by one-step Coulomb excitation from the ground state. For each state observed we measured the excitation cross section relative to that of the $2_{1}^{+}$state with an accuracy of $1-0.1 \%$. By calculating the Coulomb excitation cross sections for each excited state with the multiple-Coulomb excitation code CLX and fitting them to our experimental data (normalized to the $2_{1}^{+}$state), we deduced the electromagnetic matrix elements corresponding to each transition of the excited states. The crucial multipole-mixing ratios of the $2_{(i>1)}^{+} \rightarrow 2_{1}^{+}$transitions were obtained from $\gamma$-ray angular distributions if sufficient statistics have been obtained. A possible large $B(M 1)$ value, signature of the MSS, is then easily derived from the data. For a further description of this method, the reader is referred to Refs. $[12,13]$.

\section{Evolution of $2_{1, \mathrm{~ms}}^{+}$states in the $\mathrm{A}=\mathbf{1 3 0}$ region}

The experiments performed so far $[12,15,18,19]$ allow for a nearly complete overview on the properties of the MSS throughout the stable isotopes of the $A=130$ region. A recent publication on the first identification of a MSS in the unstable nucleus ${ }^{132} \mathrm{Te}$ [20] expands the experimental data on the $N=80$ isotonic chain to the neutron-rich

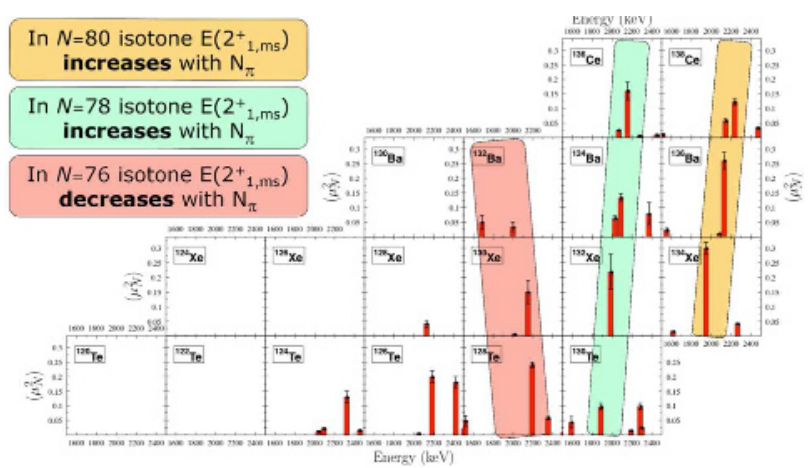

Fig. 2. Overview on the $B\left(M 1 ; 2_{i}^{+} \rightarrow 2_{1}^{+}\right)$strength distributions for the stable even-even nuclei in the $\mathrm{A}=130$ region. 
radioactive isotopes. A set of data currently under analysis on the nucleus ${ }^{132} \mathrm{Ba}$ [21] will complete our information on the one-phonon mixed-symmetry state in the stable even-even $N=76$ isotones. An overview on the $B\left(M 1 ; 2_{i}^{+} \rightarrow 2_{1}^{+}\right)$strength distributions in the even-even nuclei in the $A=130$ region is shown in figure 2 .

In the stable $N=80$ isotones the excitation energy of the $2_{1, \mathrm{~ms}}^{+}$state increases with increasing proton number. This trend continues in the unstable nucleus ${ }^{132} \mathrm{Te}[20]$. The evolution of one-quadrupole phonon MSSs along the $N=80$ isotonic chain has been studied microscopically in the nuclear shell model either using large-scale diagonalization [22] or very recently using a new importancesampling iterative algorithm for matrix diagonalization [23]. In the $N=78$ isotonic chain, the energy of the MSS again increases with increasing proton number. In the neighboring $N=76$ isotones, however, the opposite trend can be observed. Here, the excitation energy of the MSS decreases with increasing proton number. It is also interesting to follow the evolution of the MSS excitation energies in the different isotopic chains. Again, one finds that in the $\mathrm{Ce}$ and $\mathrm{Ba}$ isotopes, the excitation energy of the MSS increases with increasing neutron number, whereas in the Xe isotopes an increase in $N_{v}$ results in a decrease of $E\left(2_{1, m s}^{+}\right)$. Apparently, the $2_{1, \mathrm{~ms}}^{+}$state evolves in different ways as a function of valence particle numbers. The evolution of $M 1$ transitions between mixed-symmetry states and fully symmetric states in the $\gamma$-soft nuclei of the xenon isotopic chain have recently been described in a schematic microscopic approach [24] and in the nuclear shell model using a new importance-sampling iterative algorithm for matrix diagonalization [25]. Whether or not the observed differences are related to a critical point of a nuclear shape phase transition near ${ }^{134} \mathrm{Ba}$ is unknown up to now.

From data on $E\left(2_{1}^{+}\right)$and $E\left(2_{1, m s}^{+}\right)$, an estimate of the proton-neutron quadrupole-quadrupole interaction $V_{p n}^{Q Q}$ according to the two-state mixing scheme in [26] has been performed on the $N=80$ isotones [18], the Xe isotopes [19], and, just recently, on the $N=78$ isotones [15]. The results show, that the value derived for the proton-neutron quadrupole-quadrupole interaction in the $N=78$ isotonic chain is about $14 \%$ smaller than that for the $N=80$ isotopic chain [18] and about $6 \%$ smaller than the value for the xenon isotopic chain [19]. An example of the data, taken from Ref. [15], is displayed in figure 3.

In contrast to the lighter xenon isotopes, where the experimental sensitivity for identification of weakly collective $2^{+}$states is limited up to $2.1-2.3 \mathrm{MeV}$, for the $N=78$ isotonic chain this sensitivity extends to up to $2.5 \mathrm{MeV}$ and, in the case of ${ }^{134} \mathrm{Ba}$ [27], it extends to even higher energies. Moreover, the summed B(M1) strength remains constant as a function of proton number along the chain of $N=78$ isotones.

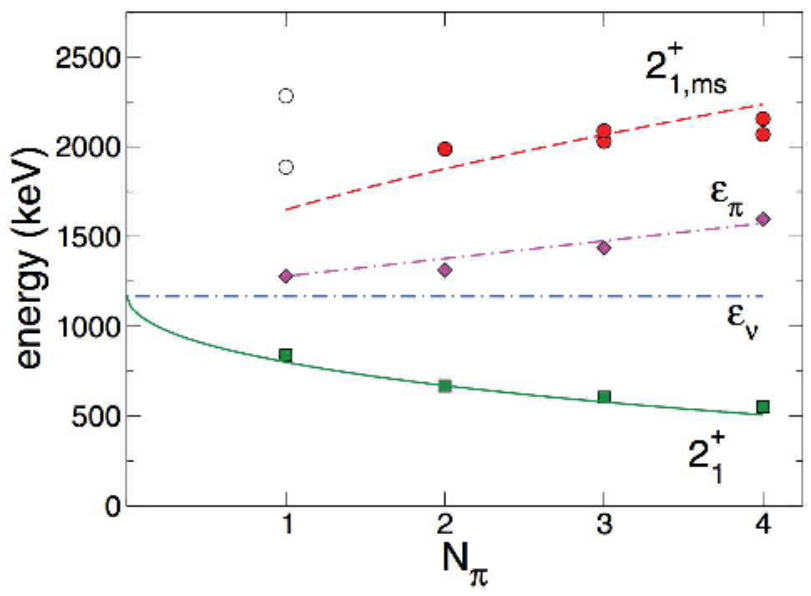

Fig. 3. Simultaneous fit of the experimental energies of the $2_{1}^{+}$ states (solid curve) and of the $B(M 1)$-weighted average energies of the $2_{1, \mathrm{~ms}}^{+}$states (dashed curve) in the $N=78$ isotones. Taken from [15].

\section{Phase of proton- and neutron- components to MSSs: the case of ${ }^{92} \mathrm{Zr}$}

We studied the formation of quadrupole collectivity in the particularly simple case of a nucleus with a low-energy structure that is dominated by one pair of valence particles each for protons and neutrons. An example is the nucleus ${ }^{92} \mathrm{Zr}$ with 2 neutrons beyond the $N=50$ shell closure and 2 protons beyond the $Z=38$ sub-shell closure. The lowest 2-quasiparticle (2qp) states will therefore have $\pi\left(1 g_{9 / 2}\right)^{2}$ and $v\left(2 d_{5 / 2}\right)^{2}$ configurations. Due to the residual proton-neutron interaction two different classes of collective excitations appear at low energy in which the amplitudes of the two most important 2qp configurations are coupled in a symmetric or antisymmetric way, respectively. In ${ }^{92} \mathrm{Zr}$, these are experimentally identified as the $2_{1}^{+}$and $2_{2}^{+}$states [13,28] with some degree of configurational isospin polarization $[29,30]$. To shed light on the microscopic origin of the effective coupling strength in the valence shell we consider the quasiparticle-phonon model (QPM) [31]. The QPM wave functions are dominated by the lowest $\pi$ and $v$ 2qp components, that show the expected in-phase and out-of-phase behavior for the $2_{\mathrm{fs}}^{+}$fully symmetric and $2_{\mathrm{ms}}^{+}$mixed-symmetry states. The electromagnetic properties and excitation energies are in excellent agreement with the data [32]. The magnetic moments of these states and the strong M1 transition between them originate almost entirely from the valence-shell configurations. However, up to $80 \%$ of the B(E2) strengths are generated from many components beyond the valence shell although their total contribution to the wave function norm is small. This observation motivates a simple three-state mixing scenario between the proton-valence shell configuration, the neutron-valence shell configuration, and the Giant Quadrupole Resonance (GQR) for a deeper insight in the formation of the one-quadrupole phonon states with symmetric and mixed-symmetry character even on a semiquantitative level [33]. For the nucleus ${ }^{92} \mathrm{Zr}$ which has 

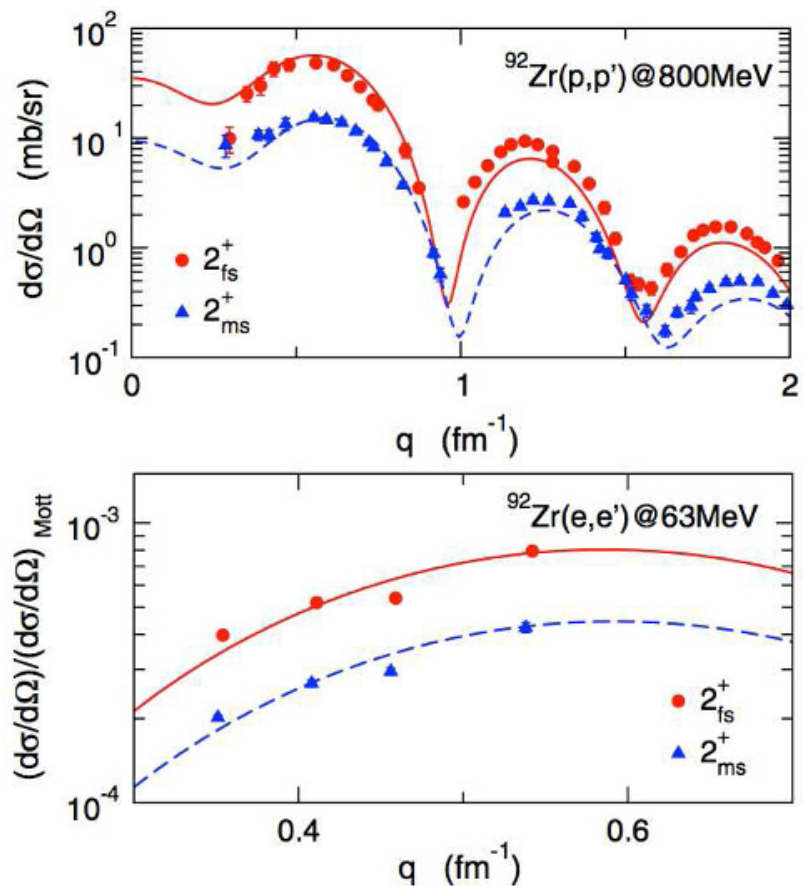

Fig. 4. Form factors for the $2_{1, \mathrm{fs}}^{+}$(red, solid line) and $2_{1, \mathrm{~ms}}^{+}$(blue, dashed line) from ${ }^{92} \mathrm{Zr}\left(p, p^{\prime}\right)$ and ${ }^{92} \mathrm{Zr}\left(e, e^{\prime}\right)$ experiments (from [33]).

a higher energy for the proton valence-shell component than the neutron valence-shell component at the $Z=40$ sub-shell closure, the three-state mixing scheme requires that the neutron valence-shell component flips its phase with respect to the GQR component when going from the proton-neutron symmetric $2_{1}^{+}$state to the predominantly mixed-symmetric $2_{2}^{+}$state.

Two probes with different sensitivity to protons and neutrons are necessary to study this quantum interference experimentally. Electron scattering at low momentum transfer provides a measure of the charge transition radius. An $\left(e, e^{\prime}\right)$ experiment was performed at the highenergy-resolution spectrometer [34] of the Darmstadt superconducting electron linear accelerator (S-DALINAC). A self-supporting zirconium metal target of $9.8 \mathrm{mg} / \mathrm{cm}^{2}$ areal density and with enrichment to $94.6 \%$ in the isotope ${ }^{92} \mathrm{Zr}$ was used. Data were taken covering a momentum transfer range between $q \sim 0.3-0.6 \mathrm{fm}^{-1}$ indicating no difference between the charge transition radii of the $2_{\mathrm{fs}}^{+}$and $2_{\mathrm{ms}}^{+}$states within experimental uncertainties (figure 4 , bottom). Information about the neutron transition radii can be derived from the proton scattering data of Ref. [35]. At the incident energy of $800 \mathrm{MeV}$ protons interact predominantly via the isoscalar central piece of the effective projectile-nucleus interaction [36]. Clearly, the refraction pattern of the $\left(p, p^{\prime}\right)$ cross section for the $2_{\mathrm{ms}}^{+}$state are shifted to higher $q$ values as compared to those for the $2_{\mathrm{fs}}^{+}$ state (figure 4, top) corresponding to a smaller transition radius.

Figure 5 displays the proton and neutron transition densities of the $2_{\mathrm{fs}}^{+}$(top) and $2_{\mathrm{ms}}^{+}$(bottom) states calculated

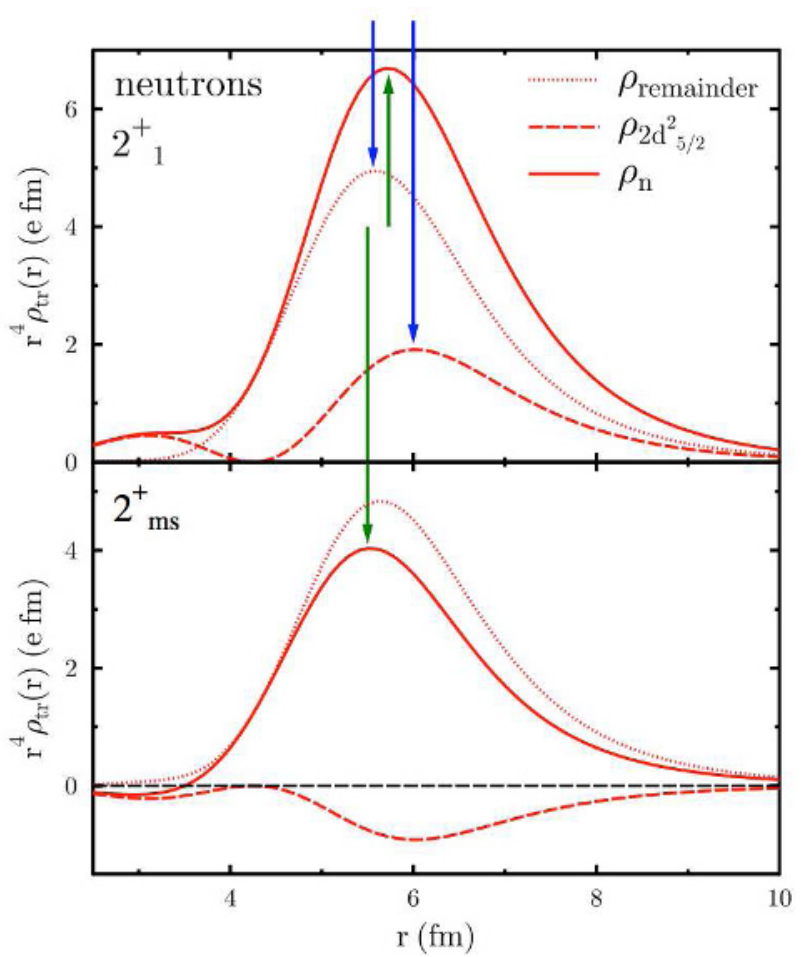

Fig. 5. Neutron transition densities of the $2_{\mathrm{fs}}^{+}$(top) and $2_{\mathrm{ms}}^{+}$(bottom) states of ${ }^{92} \mathrm{Zr}$ from QPM calculations. The full transition densities (solid lines) are decomposed in parts stemming from the GQR (dotted lines) and from the main 2qp configurations (dashed lines). The arrows indicate the maxima of the corresponding full transition densities.

in the full QPM approach. The full transition densities (solid curves) are decomposed into a collective part stemming from the GQR (dotted curves) and the predominant $v\left(2 d_{5 / 2}\right)^{2}$ ap neutron contributions (dashed curves). The key point is the different radial behaviour of both parts and their relative signs. An out-of-phase coupling between the neutron valence shell contribution and the contribution from the GQR in the $2_{1, \mathrm{~ms}}^{+}$state leads to a destructive quantum interference that reduces the neutron transition density at large radii (due to the larger radius of the $v\left(2 d_{5 / 2}\right)^{2}$ orbital) and consequently shifts the maximum of the total neutron transition density to the interior with respect to that one for the $2_{\mathrm{fs}}^{+}$state, as indicated by the arrows in figure 5 . This effect reduces the neutron transition radius of the $2_{\mathrm{ms}}^{+}$ with respect to the $2_{\mathrm{fs}}^{+}$state of ${ }^{92} \mathrm{Zr}$. In contrast, the proton transition radius remains essentially unchanged since the $\pi\left(1 g_{9 / 2}\right)^{2}$ part couples in-phase to the GQR contribution in both states. The combination of both data sets unambiguously demonstrates for the first time that the phase of the neutron valence-shell configurations in ${ }^{92} \mathrm{Zr}$ changes its sign between the $2_{\mathrm{fs}}^{+}$and the $2_{\mathrm{ms}}^{+}$state [33].

\section{Summary}

The isovector one-quadrupole phonon excitation of the valence shell has been systematically investigated in a large 
number of vibrational nuclei in the mass regions $A=90$ and $A=130$. It carries the signatures of the $2_{1, \mathrm{~ms}}^{+}$state with $F$-spin quantum number $F=F_{\max }-1$. This state is generally observed from absolute $M 1$ transition strengths when the experimental sensitivity is high enough. This state is typically concentrated in a single $2^{+}$state or distributed over two or three fragments. It is found at energies around $2 \mathrm{MeV}$ and features an $M 1$ transition matrix element to the $2_{1}^{+}$state between 0.5 and $1.5 \mu_{N}$. The details of its evolution as a function of particle number is not entirely understood. It may depend on the local shell structure around the Fermi level and on the evolution of quadrupole deformation.

\section{Acknowledgments}

We thank all those who have contributed to our research on various aspects of mixed-symmetry states. With respect to the data discussed in this contribution we particularly want to acknowledge the scientists and staff members at the Argonne National Laboratory, USA, at the iThemba Labs, South-Africa, and at the S-DALINAC, Germany and thank them for the productive scientific collaboration. Support from the DFG under grants Pi 393/2-2 and SFB 634, by the DAAD within the German-Bulgarian exchange program under grant Nos. PPP 50751591 and DNTS/01/2/2011, by the German Federal Ministry for Education and Research under grant Nos. 06DA9041I and 06DA7046, and by the Helmholtz International Center for FAIR funded through the LOEWE programme by the state of Hesse is gratefully acknowledged.

\section{References}

1. A. Arima, T. Ohtsuka, F. Iachello, and I. Talmi, Phys. Lett. B 66, 205 (1977)

2. F. Iachello, and A. Arima, The Interacting Boson Model (Cambridge University Press, 1987)

3. P. Van Isacker, K. Heyde, J. Jolie, and A. Sevrin, Ann. Phys. 171, 253 (1986)

4. D. Bohle, A. Richter, W. Steffen, A. Dieperink, N. L. Iudice, F. Palumbo, and O. Scholten, Phys. Lett. B 137, 27 (1984)

5. W. Ziegler, C. Rangacharyulu, A. Richter, and C. Spieler, Phys. Rev. Lett. 65, 2515 (1990)

6. N. Pietralla, P. von Brentano, R.-D. Herzberg, U. Kneissl, N. Lo Iudice, H. Maser, H. H. Pitz, and A. Zilges, Phys. Rev. C 58, 184 (1998)

7. T. Otsuka and K.-H. Kim, Phys. Rev. C 50, R1768 (1994)

8. N. Pietralla, C. Fransen, D. Belic, P. von Brentano, C. Frießner, U. Kneissl, A. Linnemann, A. Nord, H. H. Pitz, T. Otsuka, I. Schneider, V. Werner, and I. Wiedenhöver, Phys. Rev. Lett. 83, 1303 (1999)

9. N. Pietralla, et al., Phys. Rev. Lett. 84, 3775 (2000)

10. N. Pietralla, C. J. Barton, R. Krücken, C. W. Beausang, M. A. Caprio, R. F. Casten, J. R. Cooper, A. A. Hecht, H. Newman, J. R. Novak, and N. V. Zamfir, Phys. Rev. C 64, 031301 (2001)
11. N. Pietralla, et al., Phys. Rev. C 68, 031305(R) (2003)

12. G. Rainovski, N. Pietralla, T. Ahn, C. J. Lister, R. V. F. Janssens, M. P. Carpenter, S. Zhu, and C. J. Barton, Phys. Rev. Lett. 96, 122501 (2006)

13. N. Pietralla, P. von Brentano, and A. F. Lisetskiy, Prog.Part. Nucl. Phys. 60, 225 (2008)

14. N. Lo Iudice, C. Stoyanov, and D. Tarpanov, Phys. Rev. C 77, 044310 (2008)

15. T. Ahn, G. Rainovski, N. Pietralla, L. Coquard, T. Möller, A. Costin, R. V. F. Janssens, C. J. Lister, M. P. Carpenter, and S. Zhu, Phys. Rev. C 86, 014303 (2012)

16. I. Y. Lee, Nucl. Phys. A 520, 641c (1990)

17. P. Nolan, F. Beck, and D. Fossan, Annu. Rev. Nucl. Part. Sci. 45, 561 (1994)

18. T. Ahn, L. Coquard, N. Pietralla, et al., Phys. Lett. B 679, 19 (2009)

19. L. Coquard, et al., Phys. Rev. C 82, 024317 (2010)

20. M. Danchev, G. Rainovski, N. Pietralla, A. Gargano, A. Covello, C. Baktash, J. R. Beene, C. R. Bingham, A. Galindo-Uribarri, K. A. Gladnishki, C. J. Gross, V. Yu. Ponomarev, D. C. Radford, L. L. Riedinger, M. Scheck, A. E. Stuchbery, J. Wambach, C.-H. Yu, and N. V. Zamfir, Phys. Rev. C 84, 061306 (2011)

21. T. Möller, et al., in preparation

22. K. Sieja, G. Martinez Pinedo, L. Coquard, and N. Pietralla, Phys. Rev. C 80, 054311 (2009)

23. D. Bianco, F. Andreozzi, N. Lo Iudice, A. Porrino, and F. Knapp, Phys. Rev. C 85, 034332 (2012)

24. R.V. Jolos, N. Pietralla, N.Yu. Shirikova, and V.V. Voronov, Phys. Rev. C 84, 014315 (2011)

25. D. Bianco, F. Andreozzi, N. Lo Iudice, A. Porrino, and F. Knapp, Phys. Rev. C 84, 024310 (2011)

26. K. Heyde and J. Sau, Phys. Rev. C 33, 1050 (1986)

27. B. Fazekas, T. Belgya, G. Molnar, and A. Veres, Nucl. Phys. A 548, 249 (1992)

28. V. Werner, D. Belic, P. von Brentano, C. Fransen, A. Gade, H. von Garrel, J. Jolie, U. Kneissl, C. Kohstall, A. Linnemann, A. Lisetskiy, N. Pietralla, H. Pitz, M. Scheck, K.-H. Speidel, F. Stedile, and S. Yates, Phys. Lett. B 550, 140 (2002)

29. J. D. Holt, N. Pietralla, J. W. Holt, T. T. S. Kuo, and G. Rainovski, Phys. Rev. C 76, 034325 (2007)

30. A.P. Severyukhin, N.N. Arsenyev, and N. Pietralla, Phys. Rev. C 86, 024311 (2012)

31. V. Soloviev, Theory of Atomic Nuclei: Quasiparticles and Phonons (IoP Publ., 1992)

32. N. Lo Iudice and C. Stoyanov, Phys. Rev. C 73, 037305 (2006)

33. C. Walz, H. Fujita, A. Krugmann, P. von NeumannCosel, N. Pietralla, V. Y. Ponomarev, A. ScheikhObeid, and J. Wambach, Phys. Rev. Lett. 106, 062501 (2011)

34. A. Lenhardt, U. Bonnes, O. Burda, P. von NeumannCosel, M. Platz, A. Richter, and S. Watzlawik, Nucl. Instr. Meth. Phys. A 562, 320 (2006)

35. F. Baker, A. Scott, M. Grimm, W. Love, V. Penumetcha, C. Glashausser, G. Adams, G. Igo, G. Hoffmann, J. Moss, W. Swenson, and B. Wood, Nucl. Phys. A 393, 283 (1983) 
EPJ Web of Conferences

36. M. A. Franey and W. G. Love, Phys. Rev. C 31, 488 (1985) 